\author{
Military Technical College \\ Kobry El-Kobbah, \\ Cairo, Egypt
}

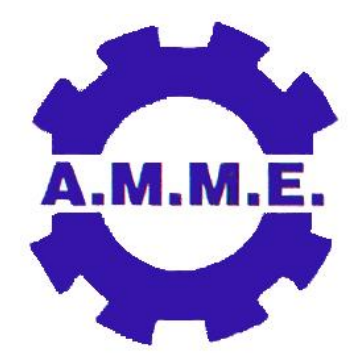

$14^{\text {th }}$ International Conference on Applied Mechanics and Mechanical Engineering AMME-14

\title{
Interactive Real-Time Driving Simulator For Vehicle Systems Design and Prototyping Process
}

By
A. M. Sharaf*
M. Sh. Asfoor*
A. Medhat*

\begin{abstract}
:
This paper presents an interactive driving simulator which is recently developed at the Military Technical College in Cairo. The simulator integrates a full vehicle model and a fully instrumented simulator cabin with the driver/researcher. The vehicle model signifies the vehicle dynamics in longitudinal, lateral and vertical directions through fourteen second-order differential equations. All necessary models for virtual representation of both the vehicle and the proving ground are carried out and integrated with the models of dynamics. The models are carried out in modular and generic forms which provide the advantage of implying the simulator for any vehicle by feeding the codes with the appropriate data. The driver reactions are measured real-time through the simulator cabin which embodies the main vehicle controllers like steering wheel, pedals and shift levers. A data acquisition system is used to digitally update the numerical models with the driver inputs. The output from simulation is instantaneously displayed via plasma screen through which the driver can monitor the vehicle motion and provide the necessary feedback. Different applications can be employed like vehicle assessment and different vehicle subsystems design e.g. suspension, drive-train system ....etc.
\end{abstract}

\section{Keywords:}

Vehicle Dynamics \& Modelling, Vehicle Performance, Driving Simulator, Virtual Reality. 
* Egyptian Armed Forces

\section{Introduction:}

Simulation of dynamic systems has been an active research area for a number of years and much progress have been carried out in the area of real-time visualization of vehicle systems in -what so called- virtual reality environment. Real-time driving simulators are very helpful in the understanding of complex interaction of the driver-vehicle during certain maneuver. Over the years, various research studies to develop low cost PC-based driving simulators with diverse applications have been carried out [1-2].

Recently, with the release of readily available and generic software packages such as Matlab/Simulink including a VRML animation toolset, a significant number of research works has been carried out, [3-4]. However, with recent demands concerning the application of control systems and Hardware-in-the-Loop (HIL) applications, there is a demand to develop less complex models, yet adequate to represent vehicle dynamics accurately. It is possible to develop such a code for a specific sub-system.

The driver response to vehicle movements is complex and yet accurately represented by driver-models. Therefore, integrating the numerical simulation and the driver is a growing demand. The basics of the modeling approach have been already presented, [5-8]. Here, the initial approach is extended by integrating the vehicle subsystems models with virtual reality models for both the vehicle and the proving ground.

The driving simulator is mainly based on a 14-degree of freedom model which simulates the different aspects of vehicle dynamics in longitudinal, lateral and vertical directions. Simulation results are illustrated real-time through a virtual animated environment includes both the vehicle and test track. The driving simulator entails a real-time calculation of vehicle dynamics in response to driver $s$ inputs such as a steering wheel, accelerator/brake pedal and gear shifting position. The simulator cabin is fully instrumented with a set of sensors which are connected to the PC through data acquisition system.

The user friendly interfaces are designed for test and calibration engineers, so no specialized knowledge is required. Both vehicle dynamics simulation and animation environment are programmed using Matlab/Simulink/Virtual Reality Toolbox which provides an open architecture permitting in-house control and simulation models to be imported into the simulation environment.

\section{The Vehicle Model}

The vehicle is divided into five masses, these are: the vehicle mass (sprung, or body mass concentrated at its centre of gravity) and located above the suspension springs, and four unsprung masses, which represent the assemblies: wheels, axles, and suspensions as shown in figure (1). The vehicle body is assumed to be rigid, with mass and moments and products of inertia. The rigid body has six DOF, which includes three translations and three rotations. 
The wheels are connected to the vehicle body via springs and dampers. It is assumed that each wheel has two DOF, one for the vertical displacement, and the other for wheel rotation . Thus, the total degrees of freedom are 14. Based on the Newton-Euler formulation, the equations of motion of the sprung mass can be written as follow [9]:

$$
\begin{gathered}
\Sigma F_{x}=m_{s} \cdot(\dot{U}-V \cdot r+W \cdot q) \\
\Sigma F_{y}=m_{s} \cdot(\dot{V}-W \cdot p+U \cdot r) \\
\Sigma F_{z}=m_{s} \cdot(\dot{W}-U \cdot q+V \cdot p) \\
\Sigma M_{x}=I_{x x} \cdot \dot{p}-\left(I_{y y}-I_{z z}\right) \cdot q \cdot r-I_{z x} \cdot(p \cdot q+\dot{r}) \\
\Sigma M_{y}=I_{y y} \cdot \dot{q}-\left(I_{z z}-I_{x x}\right) \cdot r \cdot p+I_{x z} \cdot\left(p^{2}-r^{2}\right) \\
\Sigma M_{z}=I_{z z} \cdot \dot{r}-\left(I_{x x}-I_{y y}\right) \cdot p \cdot q+I_{z x} \cdot(r \cdot q-\dot{p})
\end{gathered}
$$

For vertical dynamics, suspension forces $\left(F_{s_{i}}\right)$ are calculated based on the spring stiffness $\left(K_{i}\right)$, the shock absorber damping coefficient $\left(C_{i}\right)$ and the vertical displacement and velocity difference between the sprung mass body corner and the wheel centre as. The equation of motion for unsprung masses can be written as follow [10]:

$$
\begin{gathered}
m_{w_{i}} \cdot \ddot{z}_{w_{i}}=m_{w_{i}} \cdot g+F_{s_{i}}+F_{z_{i}} \\
m_{w_{i}} \cdot \ddot{z}_{w_{i}}=m_{w_{i}} \cdot g+\underbrace{C_{i} \cdot\left(\dot{z}_{b_{i}}-\dot{z}_{w_{i}}\right)+K_{i} \cdot\left(z_{b_{i}}-z_{w_{i}}\right)}_{\text {Suspension Force }}+F_{z_{i}}
\end{gathered}
$$

Where $\left(F_{z_{i}}\right)$ is the tire vertical force, (i) from 1:4. The effect of the vehicle body bounce $(z)$, roll angle $(\varphi)$ and pitch angle $(\theta)$ is taken into consideration, by modifying body displacement at each corner. Calculation of the wheel driving speed $\left(\omega_{i}\right)$ requires the calculation of wheel angular acceleration $\left(\dot{\omega}_{i}\right)$. From the wheel torque balance, using Newton's second law for rotational dynamics, the differential equation for the spin degree-offreedom can be written as follows [10]:

$$
I_{w_{i}} \cdot \dot{\omega}_{i}=M_{w_{i}}-M_{B_{i}}-M_{U_{i}}
$$




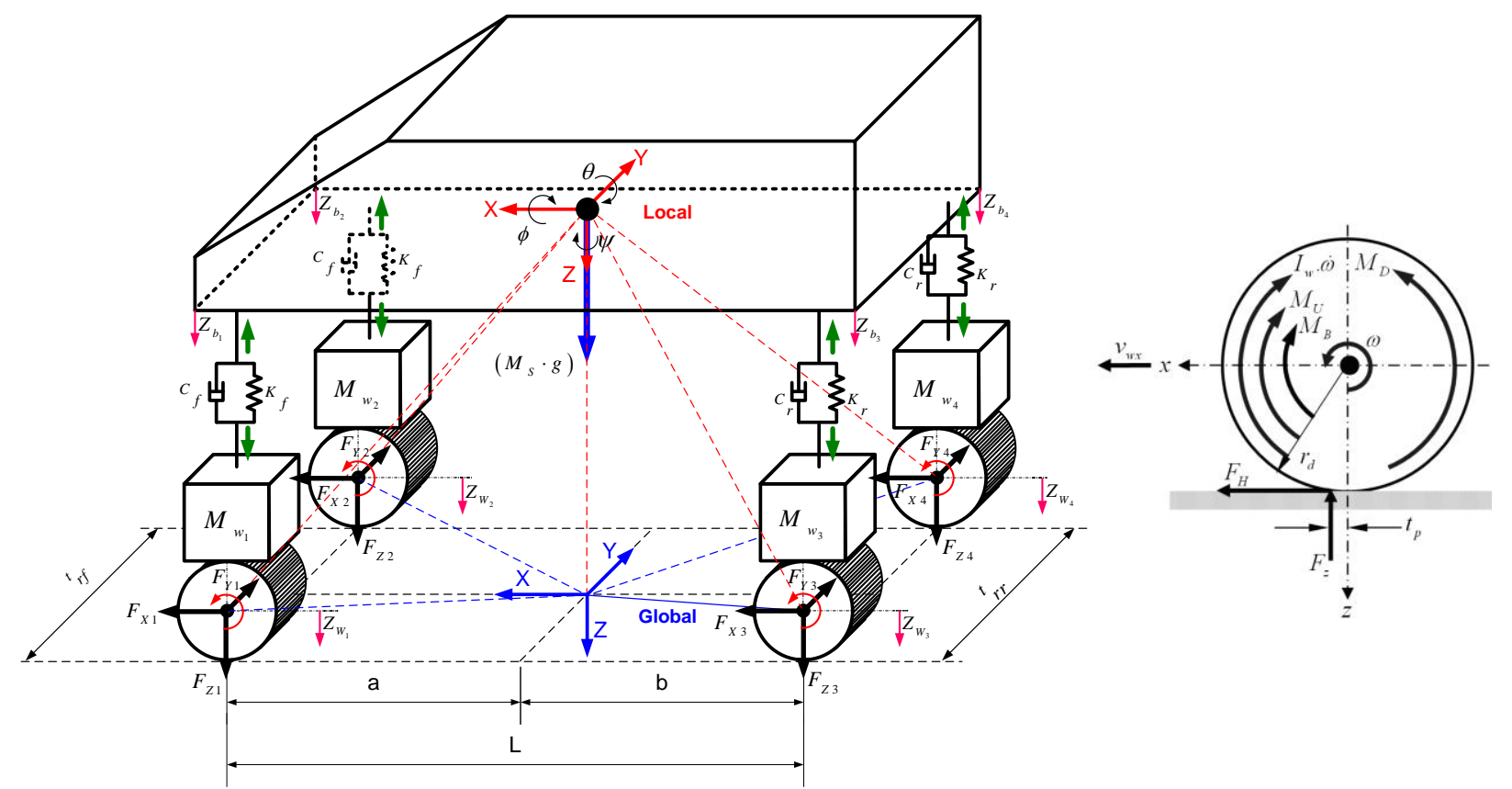

Figure (1) Free body diagram of the 14 DOF vehicle model

The drive-train model embodies the dynamic characteristics of $4 \times 4$ vehicles including the engine, clutch, a manual five-speed gear-box, and three mechanical differentials, namely: central (open/locked), front (open) and rear (open) differentials, as well as the torque transmitting elements such as propeller shafts and axles, see figure (2). The drive-train model is implemented in SimDriveline [11], which is part of the Simulink modeling, encompassing the modeling and design of systems according to the basic physical principles. More details about the derivation of the model are shown [5].

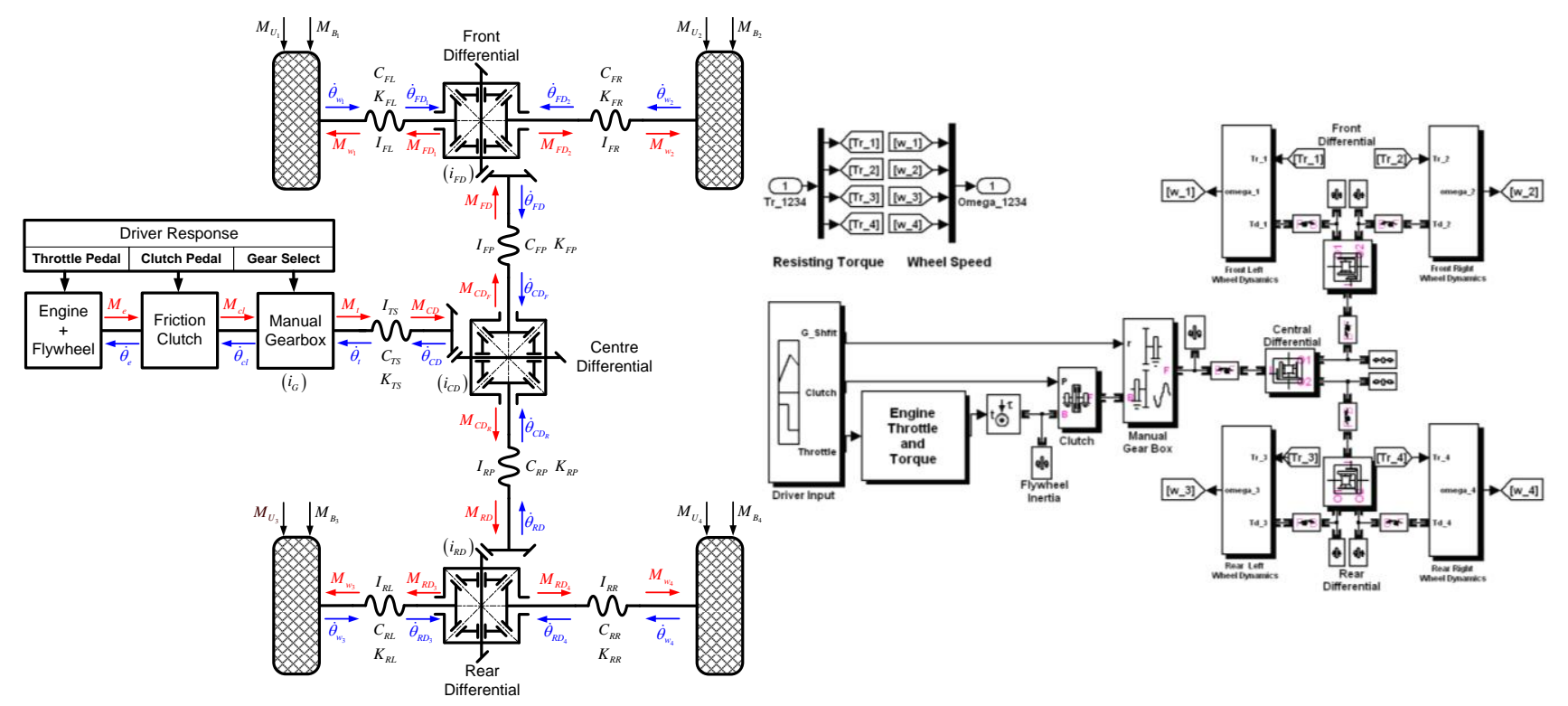

Figure (2) Mathematical Representation of $4 \times 4$ Drive-train Model 


\section{The Virtual Reality Models}

The virtual models of both the vehicle and test track are designed and implemented under the virtual reality toolbox which is launched under Matlab/Simulink [12]. The output from the vehicle dynamic models is instantaneously supplied to the VR models and simulates the motion of the vehicle. All virtual worlds have been developed using the V-Realm Builder. Figure (3) show a virtual world for both the vehicle and the test track. The VR model of the test track represents a typical track for vehicle testing to evaluate the different vehicle performance [13]. Standard tests like lane change and double lane change maneuvers can be easily performed by the simulator.
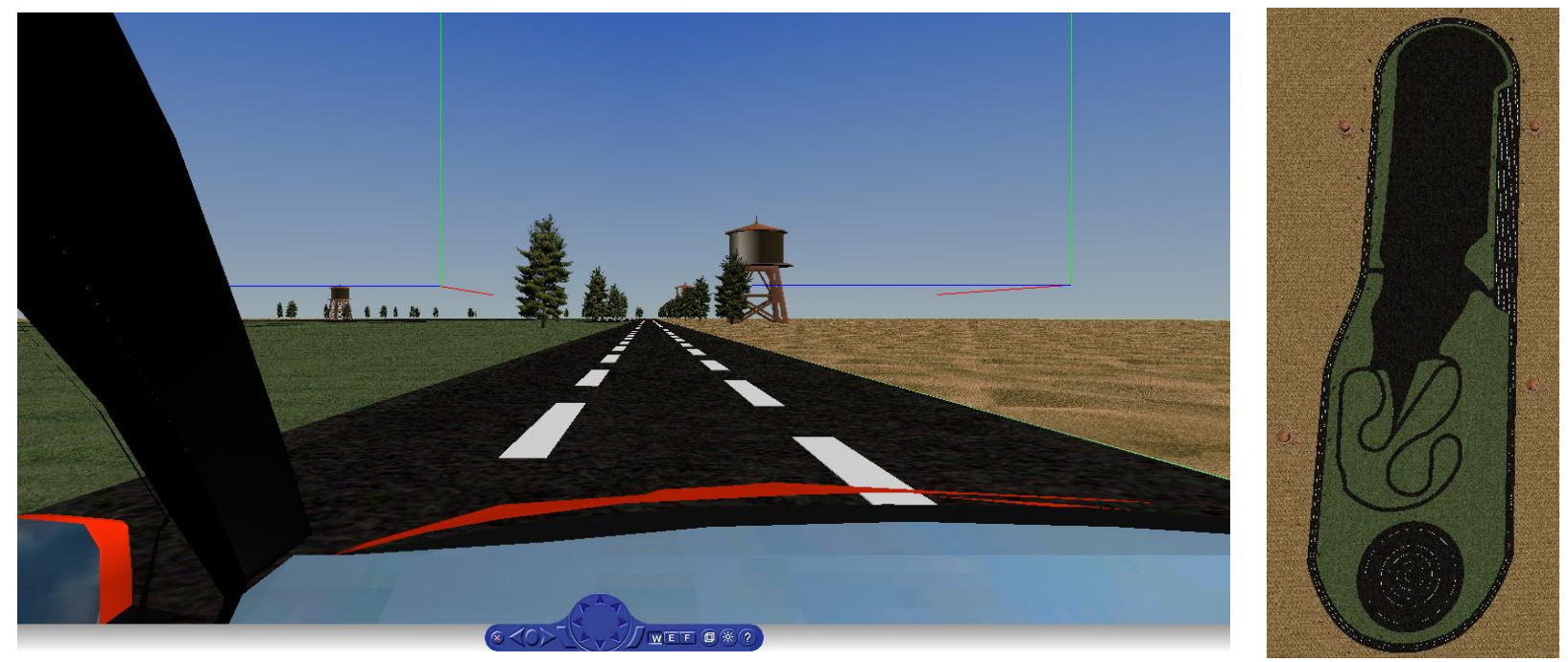

Figure (3) The Virtual 3D Models of the Vehicle and the Virtual Proving Ground

The tire forces in longitudinal, lateral and vertical directions are displayed with arrows pointing along the Cartesian coordinates. The length of each arrow is changed dynamically, representing the tire forces on the corresponding wheel. A steering wheel indicates the steering wheel angle. The results of the simulation are demonstrated real time via a flat screen and accordingly the driver responds to the simulation through the different simulator control devices.

\section{The Simulator Cabin}

The simulator cabin has typical devices from the car like accelerator pedal, brake pedal, steering wheel and shifting lever. All these devices are assembled together to enable the driver to control the motion of the car, figures (4) and (5). Each device is connected to a sensor by which the mechanical movement can be transformed to electric signal. Using a data acquisitions card, all these signals are transferred to the PC and then recalled under the environment of MATLAB to represent the input of the vehicle models. The simulation results can be illustrated as performance graphs through another screen. 


\section{Simulation Results}

As a case-study, the traction performance of a typical four wheel drive vehicle with three open differentials is investigated during a straight maneuver, figure (6). The driver increases the throttle pedal gradually till full position; then he shifts the gearbox to higher gear. During shifting, the throttle is released and the clutch is disengaged. Different traction performance measures are illustrated like the vehicle speeding up time Figure (6-a), the net tractive effort and acceleration figures (6-b) and (6-c), the saw-tooth diagram figure (6-d). It should be noted that during the shifting process and until throttle reaches its maximum position the vehicle acceleration fluctuates. This results from the instantaneous drop in vehicle speed and the effect of clutch engagement and throttle pedal application. This causes wheel slip fluctuations which affect the vehicle response and represent the real case of driving. The handling characteristics of the vehicle are shown in figure (7).

The vehicle is subjected to a double triangular steering input, applied at the front left wheel (see figure 7-a), with a constant angular speed of the steering wheel in both directions. The vehicle forward velocity is kept constant at $(65 \mathrm{~km} / \mathrm{hr})$. The variations of typical indicators for transient handling in a lane change maneuver, such as yaw velocity, lateral acceleration, and roll angle of the sprung mass with respect to time history are plotted which are used to asses vehicle stability.

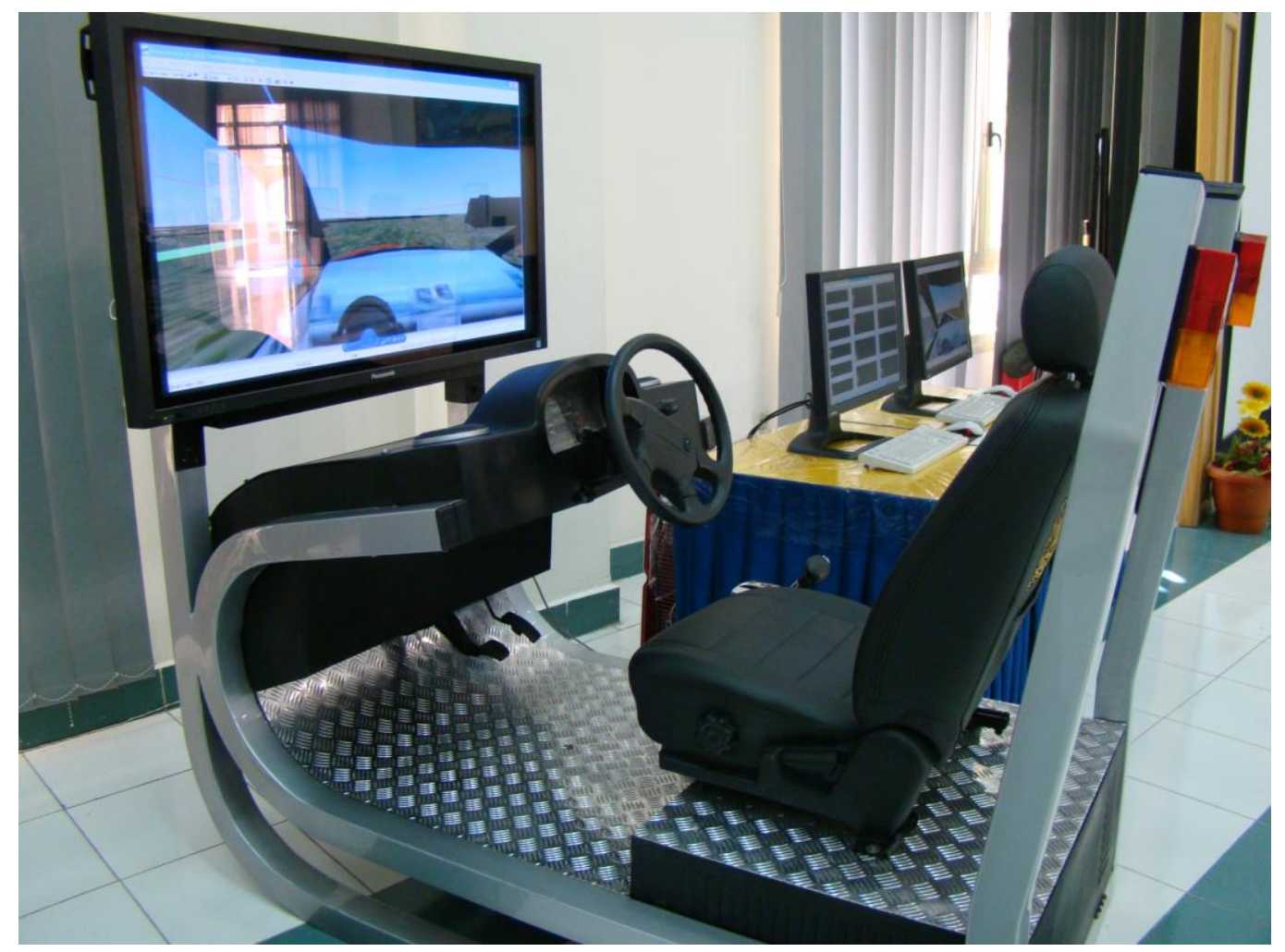

Figure (4) The Driving Simulator Developed at the Military Technical College 


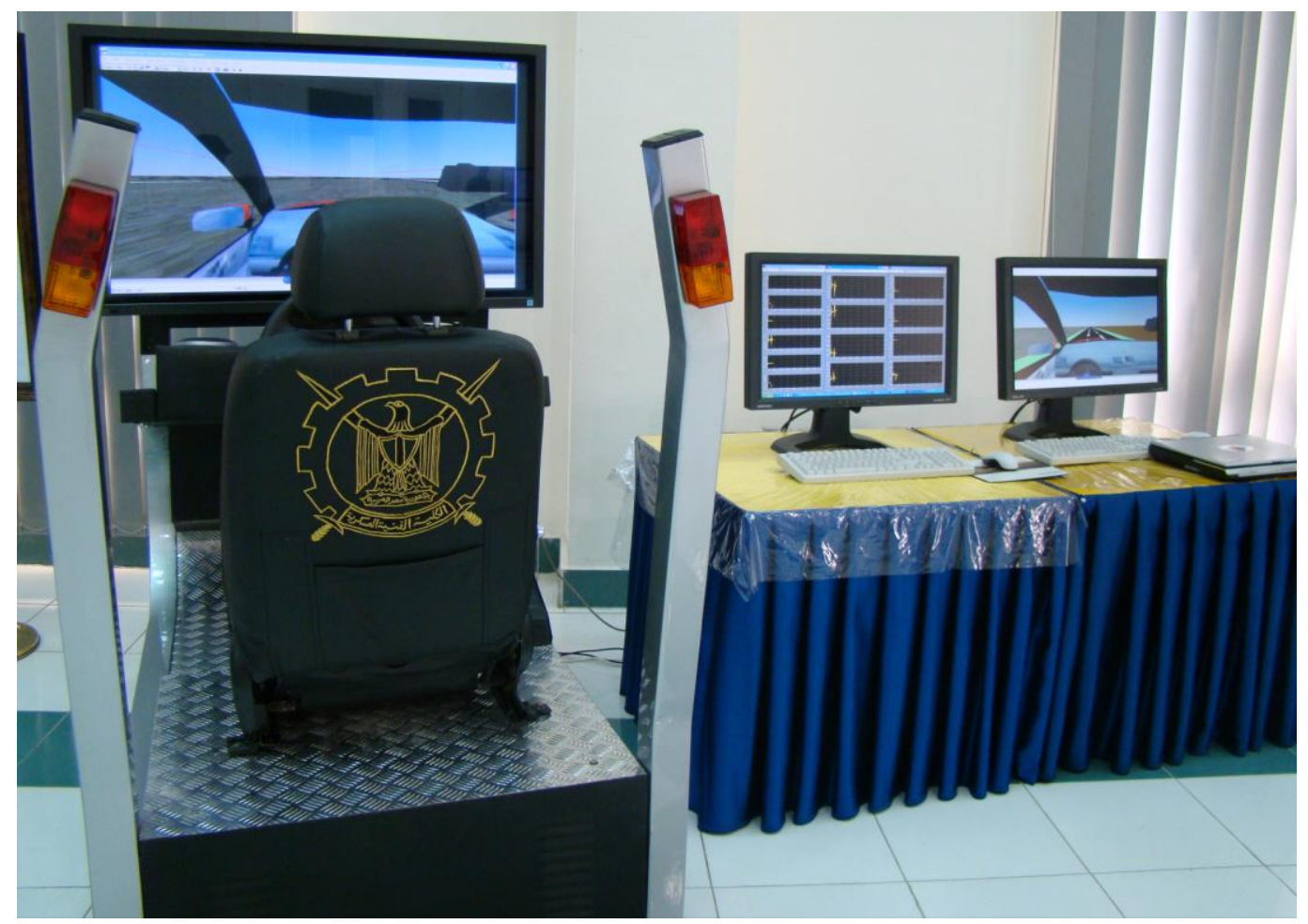

Figure (5) The Driving Simulator Developed at the Military Technical College
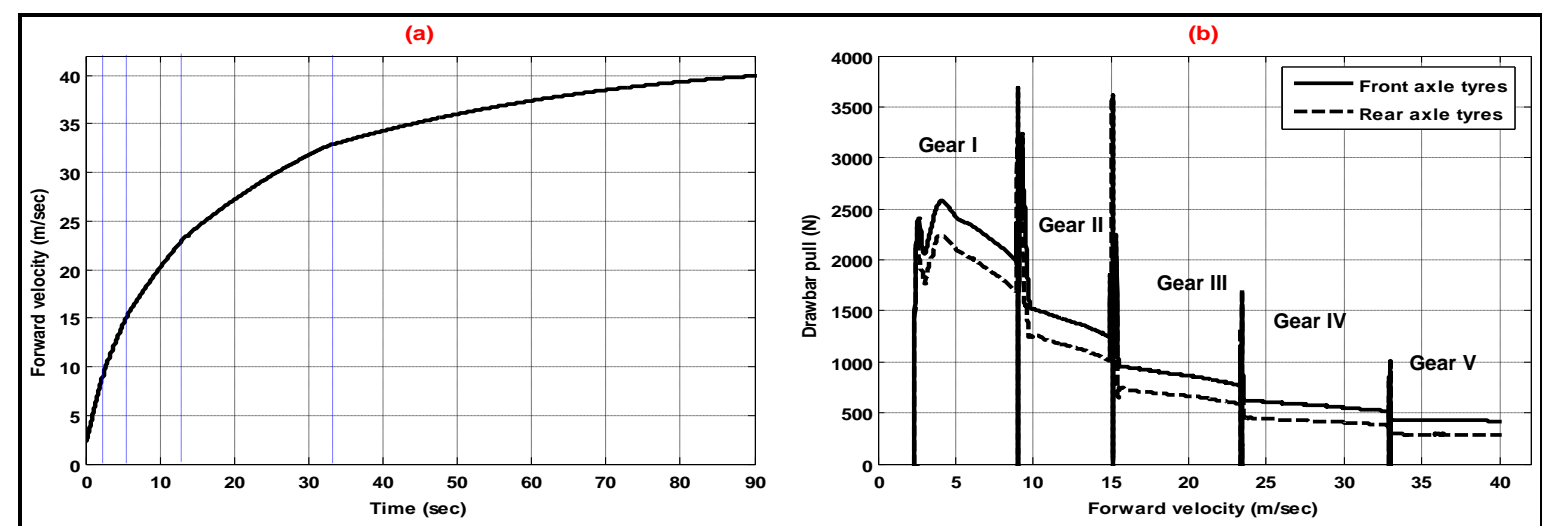

(c)

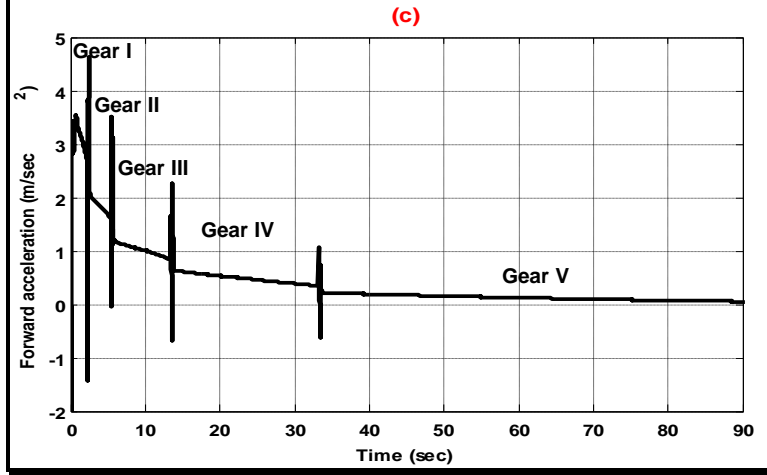

(d)

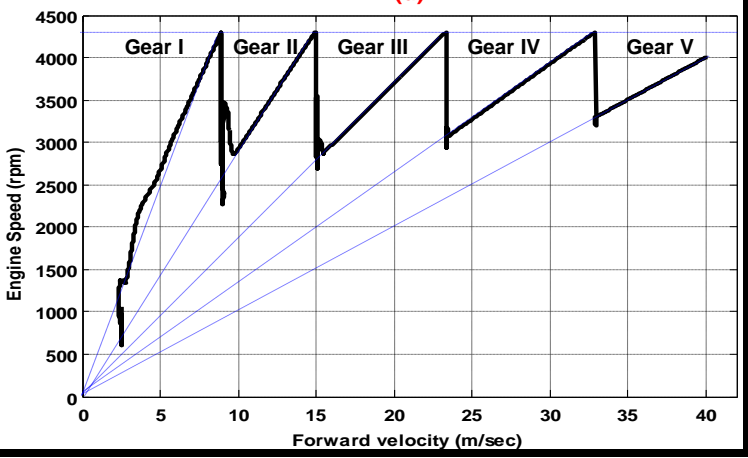

Figure (6) Traction performance During Straight Forward Maneuver 


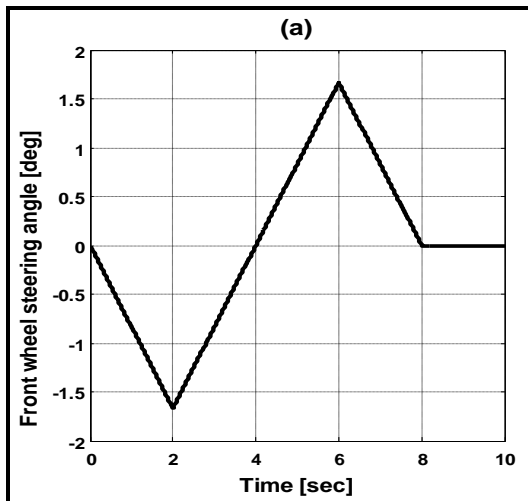

(d)

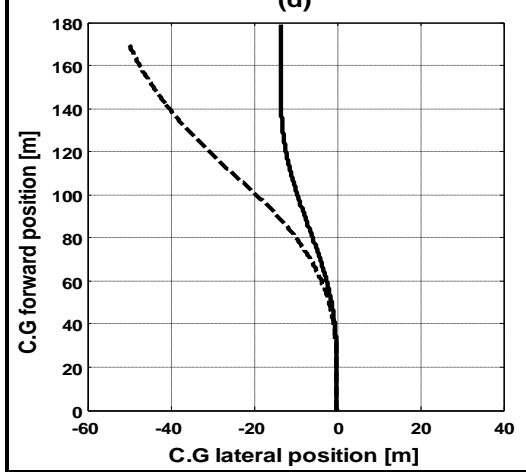

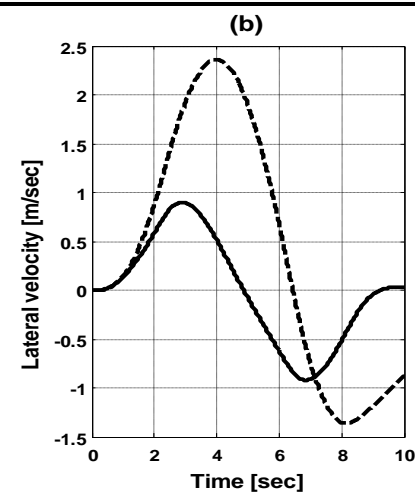

(e)

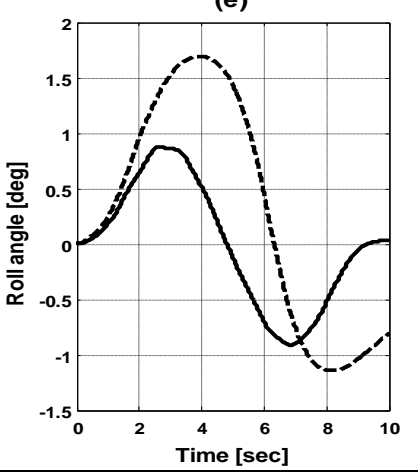

(c)

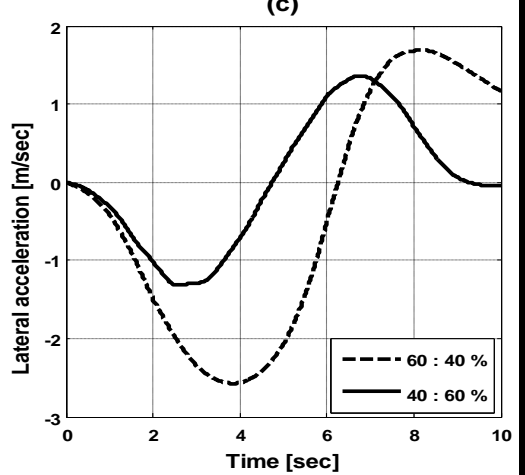

(f)

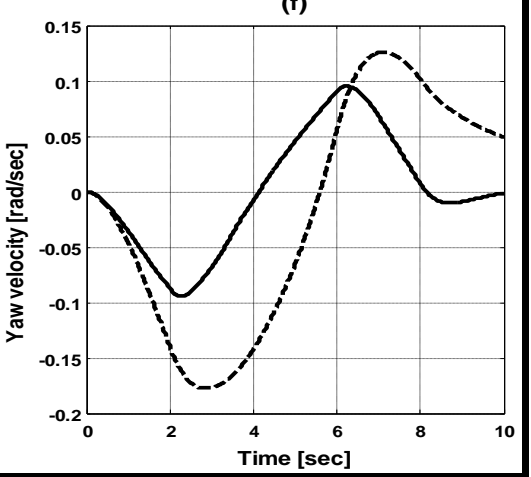

Figure (7) Transient Response during a Lane Change Maneuver

\section{Conclusions:}

A comprehensive 14-DOF vehicle model is integrated with VR models to simulate vehicle dynamics in a real time environment. Another successful integration with a hardware driving simulator including the human response is carried out. The full integration of all modules with the driver results in a driving simulator for vehicle performance evaluation. The simulator can provide the design engineers with the capability to investigate the effects of various components and aids the future development of control systems and automatic optimization of road vehicles. The models are generic and appropriate for the simulation of a wide variety of conditions, including ride and handling tests. The findings conform to natural intuition and well known published research revealing the fidelity and robustness of this simulator.

\section{References:}

[1] R. Wade Allen, George Park, Theodore J. Rosenthal and Bimal L Aponso, 'A Process for Developing Scenarios for Driving Simulations', IMAGE 2004 Conference, Scottsdale, Arizona, July 2004

[2] R. W. Allen M. L. Cook T. J. Rosenthal, 'Application of driving simulation to road safety', Advances in Transportation Studies an international Journal, 2007 Special Issue

[3] Mahesh Madurai Kumar, Taehyun Shim and Jahan Asgari, 'Development of a Vehicle Animation Tool Using Matlab Virtual Reality Toolbox', SAE Technical Paper Series No. 2007-01-0947. 
[4] Zhang Junyou and Wang Shufeng, 'The Virtual Experiment on Vehicle Handling Stability', SAE Technical Paper Series No. 2007-01-3700.

[5] A.M. Sharaf, G. Mavros, H. Rahnejat and P.D. King, "Multi-Physics Modelling Approach In All Terrain Vehicle Longitudinal Dynamics", Proceedings of IMECE06, ASME International Mechanical Engineering Congress and Exposition, Chicago, Illinois USA, November 5-10, 2006

[6] A.M. Sharaf, G. Mavros, H. Rahnejat and P.D. King and S.K. Mohan, "Direction Control and Stability of AWD Off-Road Vehicles Fitted with Visco-Lock Devices", Poster Paper, $20^{\text {th }}$ IAVSD Symposium, Dynamics of Vehicles on Roads and Tracks, 2006 Berkeley, 2007

[7] A.M. Sharaf, H. Rahnejat and P.D. King, "Analysis of Handling Characteristics of AllWheel Drive Off-Road Vehicles", Heavy Vehicle Systems, International Journal of Vehicle Design, Volume 15, Number 1/2008, Pages: 89 - 106

[8] A.M. Sharaf, G. Mavros, H. Rahnejat and P.D. King and S.K. Mohan, 'Optimisation of AWD off-road vehicle performance using visco-lock devices', Heavy Vehicle Systems, International Journal of Vehicle Design, Volume 15, Number 2-4 / 2008, Pages: 188 207

[9] Ellis, J. R. (1994) 'Vehicle handling dynamics', Mechanical Engineering Publications Limited, London, ISBN: 0852988850.

[10] Gillespie, T. D. (1992) 'Fundamentals of vehicle dynamics', Society of Automotive Engineers, Warrendale, Pa., USA, ISBN - 1560911999.

[11] The MathWorks Inc. (2004-2006) 'SimDriveline for use with Simulink user's guide version 1', http://www.mathworks.com, viewed 10 Jan 2010.

[12] Matlab Virtual Reality Toolbox 4.4, (1994-2006, The Mathworks Inc., http://www.mathworks.com, viewed 10 Jan 2010

[13] GKN Driveline Oyama Proving Ground, http://www.gkndriveline.com/drivelinecms/export/ sites/driveline/downloads/brochures/GKN ProvingGround 07 v2.pdf

\section{Nomenclatures:}

$a, b \quad$ Location of the origin of local frame of reference from front and rear axle.

$C_{f, r} \quad$ Damping coefficient of front/rear suspension.

g Gravitational acceleration.

$I_{x x}, I_{y y}, I_{z z}$ Mass moment of inertia of the sprung mass about local frame of reference.

$I_{x y}, I_{y z}, I_{z x}$ Mass product moment of inertia of the sprung mass about local frame of reference.

$I_{w i} \quad$ Mass moment of inertia of wheels.

$K_{f, r} \quad$ Stiffness coefficient of front/rear suspension spring.

$L \quad$ Wheelbase (distance between front and rear axle).

$M_{B_{i}} \quad$ Braking moment applied to each wheel. 
$M_{D_{i}} \quad$ Driving moment applied at each wheel hub.

$M_{S} \quad$ Sprung mass of the vehicle.

$M_{U_{i}} \quad$ Resisting moment applied at each wheel hub.

$M_{w i} \quad$ Unsprung mass at each wheel.

$p, q, r \quad$ Rotational velocities (roll, pitch and yaw).

$\dot{p}, \dot{q}, \dot{r} \quad$ Rotational acceleration (roll, pitch and yaw).

$R \quad$ Relative transformation matrix.

$r_{d i} \quad$ Dynamic rolling radius of each wheel.

$t_{r f}, t_{r r} \quad$ Wheel track at front and rear axle.

$U, V, W \quad$ Translational velocities (forward, lateral and vertical).

$\dot{U}, \dot{V}, \dot{W} \quad$ Translational acceleration (forward, lateral and vertical).

$z_{b i}, \dot{z}_{b i} \quad$ Vertical velocities and acceleration at corners.

$\phi, \theta, \psi \quad$ Sprung mass angular displacement (roll, pitch and yaw).

$\omega_{i}, \dot{\omega}_{i} \quad$ Wheel angular speed and acceleration. 